\title{
Interference Recognition Scheme Based on Blind Signal Separation in Satellite Communications
}

\author{
Wen Yuanyuan and Chen Hao
}

\begin{abstract}
In this paper, a novel interference recognition scheme based on blind signal separation (denoted by IRS-BSS) is studied under the satellite communication scenario. Firstly, the mixed interference signals received by the sensors are separated by using the blind signal separation (BSS) algorithm. Then, the parameters extracted from high order cumulants of interference signals are used as the classification feature vectors. Finally, using support vector machines (SVM) as classifiers, the single-tone interference, multi-tone interference, gauss noise interference and pulse interference are efficiently recognised. Simulation results demonstrate that the proposed scheme provides a very high recognition probability when SNR is not lower than $5 \mathrm{~dB}$.
\end{abstract}

Index Terms-Interference recognition, anti-jamming; support vector machines, blind signal separation, high order cumulants.

\section{INTRODUCTION}

Satellite Communication system suffers from the involuntary or intentional interference due to the exoteric communication channel. Therefore, whether civil or military systems, it is very necessary to adopt the effective anti-jamming methods. Interference detection and recognition, which have become the important steps in satellite communication, can afford the sufficient prior messages of jamming type for anti-jamming system [1]. The research of how to use the advanced technology of digital signal processing to enhance the capability of satellite communication anti-jamming is very important.

At present, the analysis to interferences is mostly detecting and restraining the special interference aiming at the special system, and the literatures about interference recognition are far less than that of interference detection. There are many methods for interference detection and suppression, such as, the technique to suppress the narrowband interference in the spread spectrum system, broadband noise interference suppression techniques based on time-frequency distributing in the spread spectrum system and so on. Using these techniques the special interference can be suppressed only and the type of the interferences cannot be automatically identified. According to [2]-[4], the high order (order>2) cumulants, which have been applied to pattern recognition, signal detection extensively, can afford more abundant

Manuscript received May 23, 2015; revised November 4, 2015.

Wen Yuanyuan is with the National Key Laboratory of Space Microwave Technology, China Academy of Space Technology, Xi'an 710100, China (e-mail: wyy031980@163.com).

Chen Hao was with the National Key Laboratory of Space Microwave Technology, China Academy of Space Technology, Xi'an 710100, China. He is now with the Department of Physics. message than the second order statistic. The most important property is that the above third order cumulants of Gaussian noise is equal to zero.

When the interference has been mixed in the satellite communication signals in time domain and frequency domain, the interference suppression is very difficult to realize. To improve the efficiency of satellite communication anti-jamming, the IRS-BSS is proposed. The structure of the paper is as follows. In Section II, we describe the course of establishing classed model of interference and the theory of the BSS. In Section III, the interference detection and recognition scheme is presented simply. Latest experimental results are showed in Section IV, and finally, Section V addresses the conclusions drawn from this work.

\section{PRINCIPLE OF THE PROPOSEd NIRS-BSS SCHEME}

The high order Cumulants provides a new method and is a powerful mathematics tool for signal processing, which describes the high order statistical characteristic of random process. Firstly, the definitions of cumulants are presented [5].

\section{A. The Basic Knowledge of the High Order Cumulants}

For an interference signal $\boldsymbol{J}(t)$, the high order cumulants can be defines as:

$$
\begin{gathered}
\boldsymbol{C}_{21}=\operatorname{Cum}\left(J, J^{*}\right)=\boldsymbol{M}_{21} \\
\boldsymbol{C}_{42}=\operatorname{Cum}\left(J, J, J^{*}, J^{*}\right)=\boldsymbol{M}_{42}-\boldsymbol{M}_{20} \boldsymbol{M}_{22}-2 \boldsymbol{M}_{21}{ }^{2} \\
\boldsymbol{C}_{63}=\operatorname{Cum}\left(J, J, J, J^{*}, J^{*}, J^{*}\right)=\boldsymbol{M}_{63}-9 \boldsymbol{C}_{42} \boldsymbol{M}_{21}-6 \boldsymbol{M}_{21}{ }^{3} \\
\boldsymbol{C}_{80}=\boldsymbol{M}_{80}-28 \boldsymbol{M}_{20} \boldsymbol{M}_{60}-35 \boldsymbol{M}_{40}{ }^{2}+420 \boldsymbol{M}_{40} \boldsymbol{M}_{20}{ }^{2}-630 \boldsymbol{M}_{20}{ }^{4} \\
\boldsymbol{C}_{84}=\boldsymbol{M}_{84}-16 \boldsymbol{C}_{63} \boldsymbol{M}_{21}-\left|\boldsymbol{C}_{40}\right|^{2}-18 \boldsymbol{C}_{42}{ }^{2}-72 \boldsymbol{C}_{42} \boldsymbol{M}_{21}{ }^{2}-24 \boldsymbol{M}_{21}{ }^{4}
\end{gathered}
$$

where $\boldsymbol{M}_{p q}$ denotes $p$-order hybrid moment of signal, with the definition given by

$$
\boldsymbol{M}_{p q}=E\left[\boldsymbol{J}(t)^{p-q} \boldsymbol{J}^{*}(t)^{q}\right]
$$

\section{B. Feature Parameters Extraction}

The high order cumulants of the signal embody the 
information of the signal type, which is different when the high order cumulants are different. The high order cumulants can be regarded as a sign of the signal type, and through validating the sign the signal type can be identified [6].

Supposing that the sending code element sequences are independent identically distributed. In the ideal circumstances, the high order cumulants of the interference signals are calculated by $(1) \sim(6)$. In order to realize the identification of interference signals, we can use different combinations of cumulants to set up identification parameters. We extract some feature parameters from the high order cumulants to identify the interference type [7]. Feature parameter vectors of the interference recognition are given by:

$$
\boldsymbol{F}_{s}=\left[F_{1}, F_{2}, F_{3}\right]=\left[\frac{\left|\boldsymbol{C}_{63}\right|^{2}}{\left|\boldsymbol{C}_{42}\right|^{3}}, \frac{\left|\boldsymbol{C}_{42}\right|}{\left|\boldsymbol{C}_{21}\right|^{2}}, \frac{\left|\boldsymbol{C}_{80}\right|}{\left|\boldsymbol{C}_{84}\right|}\right]
$$

where $\boldsymbol{C}_{p q}$ stands for the high order cumulants of the interference signals, and can be calculated by $(1) \sim(6)$, $p, q$.

\section{BSS Algorithm Based on the Householder Transformation}

Let $S=\left\{N_{r} \mid 1 \leq r \leq s\right\}$ be a set of $S$ matrices with common size $n \times n$. A joint diagonalizer based on householder transformation of the set $\boldsymbol{S}$ is defined to minimize the following criterion [8]

$$
C(\boldsymbol{H}, \boldsymbol{S}) \stackrel{\text { def }}{=} \arg \min \sum_{r=1, \ldots, s}\left\|\boldsymbol{N}_{r}-\operatorname{diag}\left(\boldsymbol{N}_{r}\right)\right\|_{F}^{2}
$$

where $\operatorname{diag}(\cdot)$ is an operator that zeros the off-diagonal elements of a matrix. \|\|$_{F}^{2}$ is the squared Frobenius norm. When the set $\boldsymbol{S}$ contains only one hermitian matrix, joint diagonalization is equivalent to usual unitary diagonalization. If the set $\boldsymbol{S}$ cannot be exactly jointly diagonalized (this is the case when sample cumulants are processed), the unitary minimization of (8) defines a somewhat arbitrary but quite natural 'joint approximate diagonalization'.

A square matrix of the form $H=I-2 w w^{T}$, where $\|w\|_{2}=1$, is defined to be a Householder transformation. A vector $\mathbf{x}$ may be transformed by a Householder transformation to a vector with each element zero except for the first, i.e.

$$
H x=r e_{j} ; \quad r \neq 0
$$

Say, where $r$ is a constant and $e_{j}$ is a vector with each component 0 except for the $\mathrm{j}$-th which is $1(j=1,2, \ldots, n)$.

\section{THE PROPOSED NIRS-BSS SCHEME}

It is very difficult to select the feature vectors of the mixed interferences, because the feature vectors have been changed when the interferences mixing. If we want to use the SVM algorithm to identify the mixed interference signals, a natural thought is to separate the mixed interference signals firstly. The BSS algorithm is adopted to separate the mixed interference signals.

In the BSS, multiple independent source signals are extracted from their linear mixtures with little to no knowledge of the sources and the mixing system [9]. The technology of BSS has been widely used in many fields, such as radar, speech signal processing and so on. Many algorithms have been proposed for the BSS problem. In the paper the BSS Algorithm based on the Householder transformation has been employed to separate the mixed interferences.

The algorithm block diagram is shown in Fig. 1. It can be seen from Fig. 1 that the algorithm is composed of three main components: the interference model establishing, the separation of interference and the interference recognition. In the first module, we will simulate the four interferences and extract the feature parameters of interferences; we will train the feature vectors using SVM and store the feature vectors in database for establishing the interference model. In the second module, the received signals will be separated using the algorithm of BSS. In the third module, the type of separated interferences will be recognized contrasting the interference model.

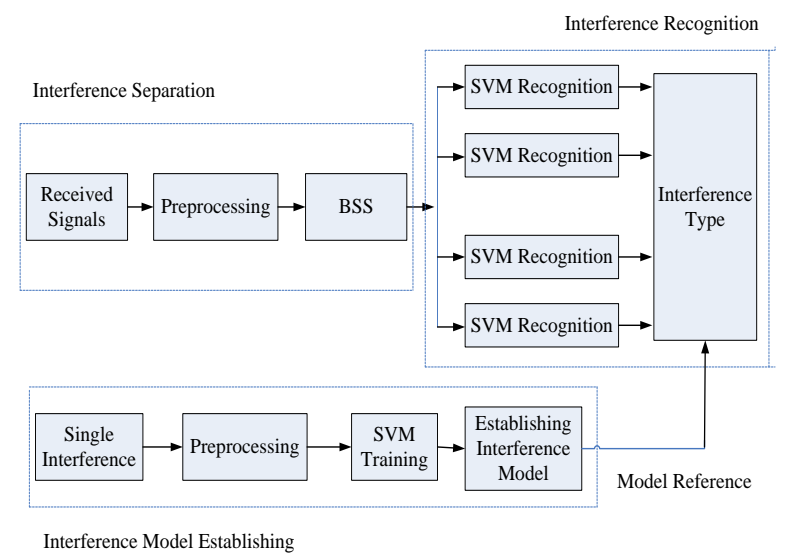

Fig. 1. Block diagram of the proposed NIRS-BSS scheme.

\section{Simulation Results}

In this paper, we will place emphasis on the familiar interference types such as gauss noise interference (GNI), single-tone interference (STI), multi-tone interference (MTI) and pulse interference (PI). Single-tone, multi-tone and gauss noise interference are chosen because they are very common and simple in an aactual communication system. And pulse interference is a kind of broad band interference which can affect the performance of many communication systems, so we choose it as another kind of interference.

In this section, the simulation of the interference signals identification which includes STI, MTI, GNI and PI is finished according to the Section III. The additive noise is the complex white Gaussian.

The four original interferences are received by four sensors. We adopt the BSS Algorithm based on the Householder transformation to separate the mixed interference signals and the result is shown in Fig. 2. The first column of Fig. 2 depicts the four original interference signals, which are STI, MTI, GNI and PI in order. The second column plots the mixed 
interference signals which are received by sensors. We cannot get any useful information from the mixed interference signals, so the traditional recognition algorithms cannot work in this situation. The third column shows the separation result, which is the estimation of the original interference signals. The order of the four interferences has been changed, and we can adopt the SVM algorithm to identify the interference types.
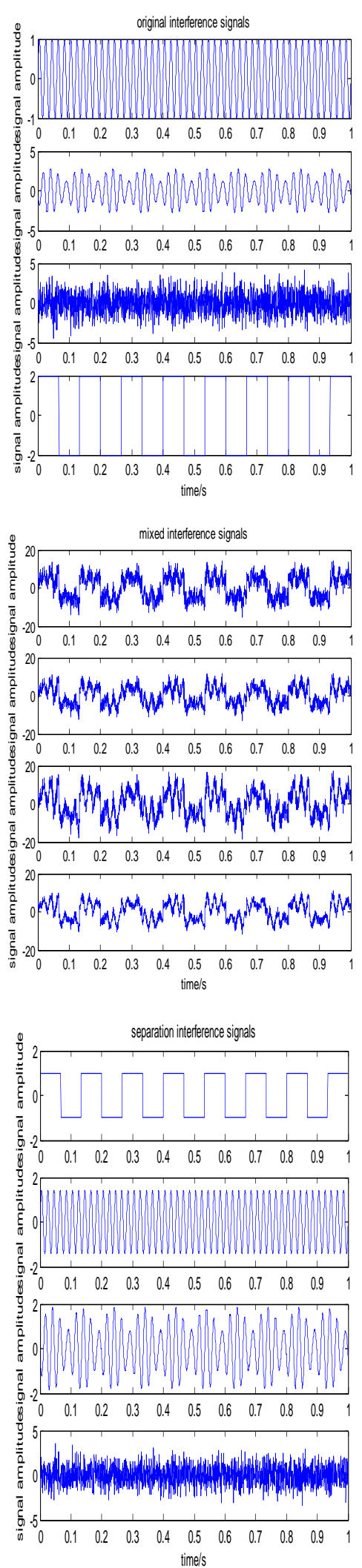

Fig. 2. Original, mixed and separation interference signals.

\section{A. Variety of the Feature Parameters versus the SNR}

To distinguish four interference types, simulation runs were carried out with 2400 samples. When the SNR ranging from $0 \mathrm{~dB}$ to $20 \mathrm{~dB}$, the 100 samples selected is simulated, and the feature parameters of which will change following the SNR. Fig. 3, Fig. 4, and Fig. 5 plots the change of the feature vectors mean of 100 samples versus SNR.
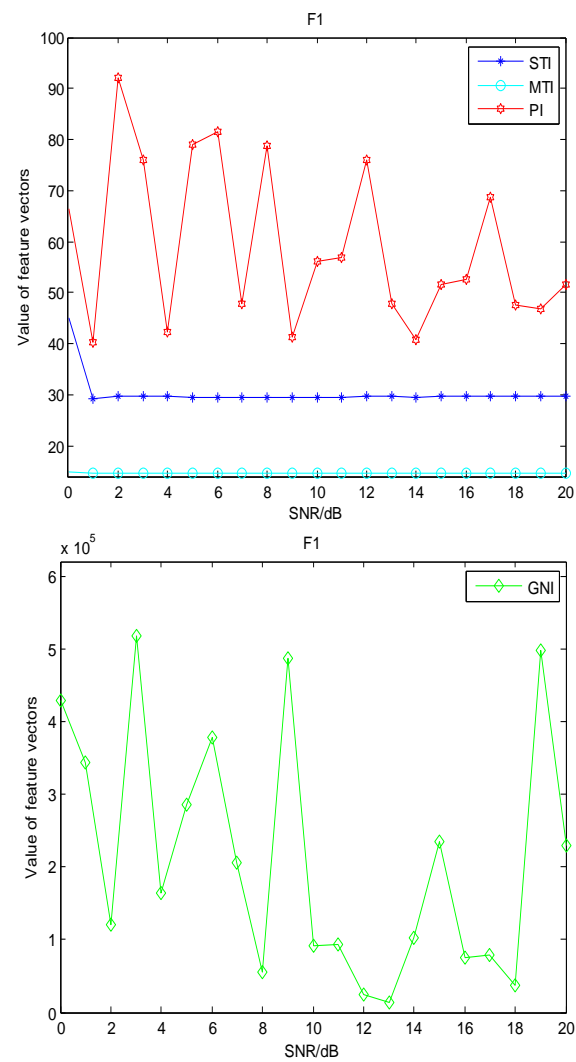

Fig. 3. $F_{1}$ versus SNR

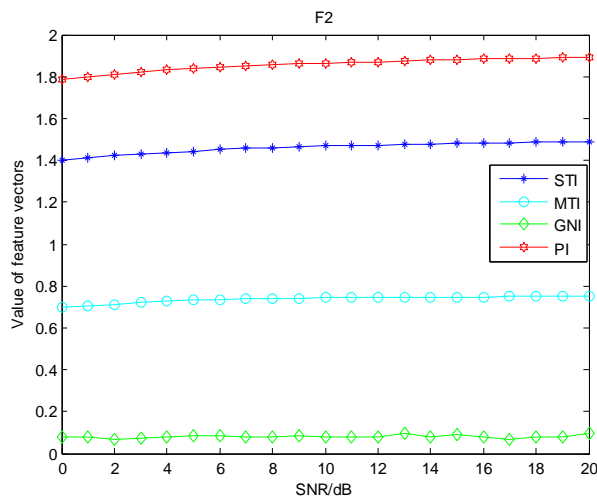

Fig. 4. $F_{2}$ versus SNR.

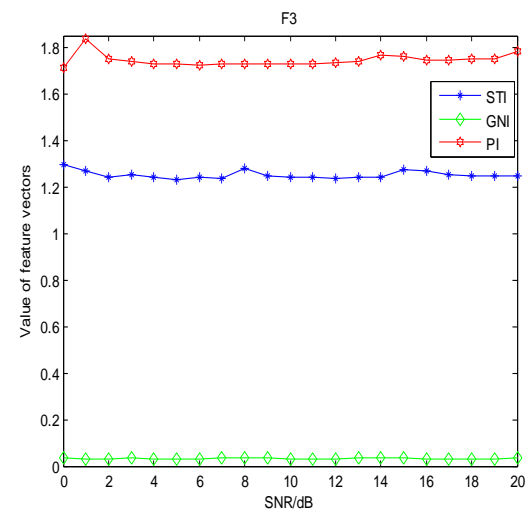




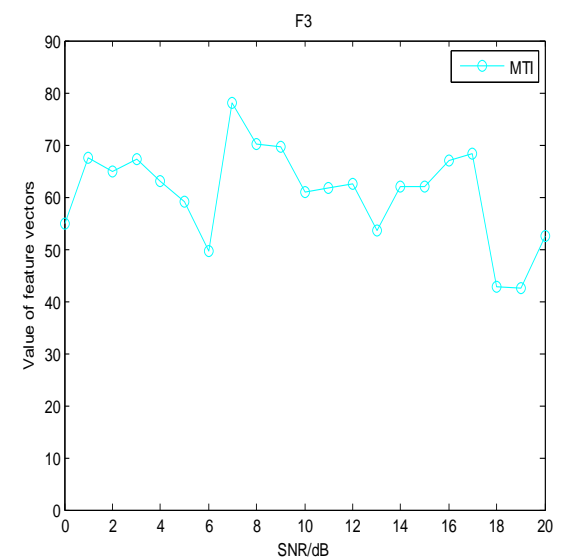

Fig. 5. $F_{3}$ versus SNR.

It can be shown that there are the different feature vectors for the different interferences from the above figure. So the four interferences can be recognized through the three feature parameters, which provide a basis to SVM recognition.

\section{B. Variety of the Recognition Probability versus the SNR}

Respectively at $0 \mathrm{~dB}, 5 \mathrm{~dB}, 10 \mathrm{~dB}, 15 \mathrm{~dB}$ and $20 \mathrm{~dB}$, the 600 samples selected from each signal are simulated, 500 samples of which for training, 100 samples of which for testing. And the total length of each sample is 2400 points. The recognition probability is shown in Table I.

TABLE I: RECOGNITION PROBABILITY BASED ON THE PROPOSED NIRS-BSS SCHEME

\begin{tabular}{|c|c|c|c|c|c|}
\hline \multirow{2}{*}{$\begin{array}{c}\text { Interference } \\
\text { signals }\end{array}$} & \multicolumn{5}{|c|}{ Recognition Probability (\%) } \\
\cline { 2 - 6 } & $0 \mathrm{~dB}$ & $5 \mathrm{~dB}$ & $10 \mathrm{~dB}$ & $15 \mathrm{~dB}$ & $20 \mathrm{~dB}$ \\
\hline STI & 94 & 97 & 98 & 98 & 99 \\
\hline MTI & 100 & 100 & 100 & 100 & 100 \\
\hline GNI & 97 & 98 & 98 & 98 & 98 \\
\hline PI & 84 & 90 & 93 & 95 & 95 \\
\hline
\end{tabular}

From the above analysis, the mentioned NIRS-BSS scheme can recognize the familiar interference correctly in communication system under low SNR. When SNR exceeds $5 \mathrm{~dB}$, all recognition probability will exceed $90 \%$.

\section{CONCLUSION}

Satellite communication system requires strong anti-jamming ability. A new method is presented which combined SVM of the signals' high order cumulants as feature vectors and BSS Algorithm based on the Householder transformation to identify the four interference signals without prior information in this paper. We investigate the performance of the proposed scheme using numerical simulation. Simulation results show that the method can recognize interferences correctly at low SNR. Recognition probability can achieve over the $90 \%$ at the SNR of $5 \mathrm{~dB}$.It can afford the sufficient prior messages of interferences for satellite communication system and ensure us succeed in adopting the efficient anti-jamming methods rapidly.

\section{REFERENCES}

[1] L. Kong, Z. J. Xu, J. M. Wang, and K. X. Pan, “A Novel algorithm for jamming recognition in wireless communication," CISP 2013, vol. 6, no. 10 , pp. 1473-1477, 2013.

[2] M. R. Mirarab, and M. A. Sobhani, "Robust modulation classification for PSK/QAM/ask using higher-order cumulants," in Proc. the 6th ICICS, vol. 3, no.12, pp. 58-64, 2007.

[3] E. H. Jayatunga and O. A. Dobre, "A robust higher-order cyclic cumulants feature-based vector for QAM classification," CSNDSP 2014, vol. 9, no. 8, pp. 417-421, 2014.

[4] Z. J. Xu, J. M. Wang, and L. Kong, "A Novel method for jamming recognition based on multi-slices of cyclic spectrum density," in Proc. ICMSEEC 2013, vol. 8, no. 11, pp. 1135-1139, 2013.

[5] C. L. Nikias and A. P. Petropulu, "Higher-order spectra analysis," Ptr Prentice Hall, Englewood Cliffs, New Jersey, 1993.

[6] L. X. Wang, and Y. J. Ren, "Recognition of digital modulation signals based on high order cumulants and support vector machines," in Proc. 2009 ISECS, vol. 1, no. 9, pp. 271-274, 2009.

[7] M. Z. Liu, Y. Zhao, L. Shi, and J. W. Dong, "Research on recognition algorithm of digital modulation by higher order cumulants," in Proc. ICIMCCC2014, vol. 4, no. 1, pp. 686-690, 2014.

[8] C. Rader and A. Steinhardt, "Hyperbolic householder transformations," IEEE Trans. Acoust., Speech, Signal Proc., vol. 8, no. 4, pp. 1-5, 1988.

[9] K. Rahbar, and P. James, "A frequency domain method for blind source separation of convolutive audio mixtures," IEEE Transactions on Speech and Audio Processing, vol. 3, no. 5, pp. 1-13, 2005.

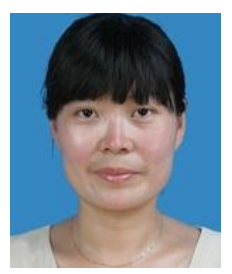

Wen Yuanyuan was born in 1980. She got her Ph.D degree and an engineer. Her mainly worked at blind signal separation and anti-jamming communication theory and technology. 